\title{
Localized Lasing Mode in GaN Quasi-Periodic Nanopillars at Room Temperature
}

\author{
Tzeng-Tsong Wu, Student Member, IEEE, Chih-Cheng Chen, Hao-Wen Chen, Tien-Chang Lu, Senior Member, IEEE, \\ Shing-Chung Wang, Life Member, IEEE, and Cheng-Huang Kuo
}

\begin{abstract}
In this paper, GaN quasi-periodic nanopillars were fabricated and investigated. The quasi-periodic nanopillars were realized by nanoimprint technique and selective area growth. Localized lasing mode was identified in the GaN quasi-periodic nanopillars. The threshold energy density and lasing wavelength were $40 \mathrm{~mJ} / \mathrm{cm}^{2}$ and $369 \mathrm{~nm}$, respectively. The divergence angle and near-field lasing spot were measured to be $10.5^{\circ}$ and $3.6 \mu \mathrm{m}$, respectively. The spontaneous emission coupling factor of localized lasing mode was estimated to be $9.4 \times 10^{-3}$. The mode patterns in the real and reciprocal spaces were calculated by the multiple scattering method to confirm the mode localization behavior.
\end{abstract}

Index Terms-GaN, mode localization, nanopillar.

\section{INTRODUCTION}

$\mathbf{P}$ HOTONIC crystals (PCs), incorporated of periodic structures with its unique optical properties, have been widely adopted to many optoelectronic devices, including lightemitting diodes (LEDs) and PC lasers [1]-[13]. Two kinds of PC lasers-band-edge lasers [3]-[11] and defect-type lasers [12], [13] — have been proposed and investigated during the past decade. For the band-edge PC lasers, the specific Bragg diffraction could occur at the photonic band edges to achieve surface-emitting condition and laser oscillation in a large area by controlling the period and lattice of PCs. This type of PC lasers, called PC surface-emitting lasers (PCSELs), could be potential for display and high-power laser application. PCSELs has been explored in many different wavelength regions [6]-[11]. On the other hand, most of the defect-type PC lasers with a thin membrane suspended in the air have been realized in InP and GaAs material systems due to the easy removal of underlying sacrificial layers. Due to photonic bandgap effect in the in-plane direction and total internal reflection in the vertical direction to the thin membrane, highly localized laser oscillations

Manuscript received November 1, 2012; revised January 24, 2013; accepted February 4, 2013. Date of publication March 27, 2013; date of current version May 13, 2013. This work was supported in part by the Ministry of Education Aim for the Top University program and in part by the National Science Council of Taiwan under Contract NSC98-2923-E-009-001-MY3 and Contract NSC992622-E009-009-CC3.

T.-T. Wu, C.-C. Chen, H.-W. Chen, T.-C. Lu, and S.-C. Wang are with the Department of Photonics, Institute of Electro-Optical Engineering, National Chiao Tung University, Hsinchu 30050, Taiwan (e-mail: mulderbob.eo98g@nctu. edu.tw; tms24028.eo97g@g2.nctu.edu.tw; Chenemmaemmachen@gmail.com; timtclu@mail.nctu.edu.tw; scwang@cc.nctu.edu.tw).

C.-H. Kuo is with the Institute of Lighting and Energy Photonics, National Chiao Tung University, Tainan 711, Taiwan (e-mail: kuoch@ @ail.nctu.edu.tw).

Color versions of one or more of the figures in this paper are available online at http://ieeexplore.ieee.org.

Digital Object Identifier 10.1109/JSTQE.2013.2247570 can be observed in the defect cavities to achieve with special properties [12], [13]. These special properties such as small modal volume and localized lasing mode are important for development of ultralow threshold lasers and photonic integrated circuits. However, it is rather difficult to fabricate suspended membrane structures in GaN-based materials by selective etching due to their stable chemical bonding properties [14], [15]. On the other hand, a quasi-periodic structure, named photonic quasi-crystals (PQCs), is highly rotational symmetric in the reciprocal space and capable of exhibiting photonic bandgaps for mode localization [16], [17]. Recently, several numerical studies in PQCs, organic lasers with Penrose quasi-crystals (QCs) and waveguide device with 12 -fold QCs have been realized and demonstrated [18]-[22]. It suggests that quasi-periodic structures could be a good candidate to demonstrate localized laser oscillation in a nonmembrane structure.

In this paper, we have fabricated and demonstrated localized lasing mode in GaN quasi-periodic nanopillars. In Section II, the fabrication process of GaN quasi-periodic nanopillars including nanoimprint lithography (NIL) and selective area growth (SAG) will be described. Moreover, the optical pumping system will also be introduced. In Section III, the lasing characteristics including input-output characteristics, lasing spectra, polarization, and divergence angle obtained under the optical pumping condition at room temperature will be presented. A localized lasing mode in GaN quasi-periodic nanopillars with small field distribution in the UV region was observed in the near-field image. In Section IV, we further utilize multiple scattering method and plane-wave expansion (PWE) method to calculate the mode pattern of $\mathrm{GaN}$ quasi-periodic nanopillars in real and reciprocal space to confirm the experimental results. The results show that the localized mode would be confined in the quasi-periodic nanopillars. Finally, a summary of these results will be described in Section V.

\section{FABRICATION AND EXPERIMENT}

The GaN quasi-periodic nanopillars used in this study were all prepared by metal-organic chemical vapor deposition (MOCVD). We first deposited a low-temperature (LT) GaN nucleation layer and a 2- $\mu \mathrm{m}$-thick undoped $\mathrm{GaN}$ layer on a c-face sapphire substrate. A 60-nm-thick $\mathrm{SiO}_{2}$ film was then deposited on the GaN surface by plasma-enhanced chemical vapor deposition (PECVD). A quasi-periodic nanohole array was subsequently formed as growth masks on the GaN surface using NIL and dry etching. The sample was then sent to MOCVD for the nanopillar growth. At the starting of $\mathrm{GaN}$ nanopillar growth, the growth temperature was $990^{\circ} \mathrm{C}$ at the chamber pressure of 
(a)
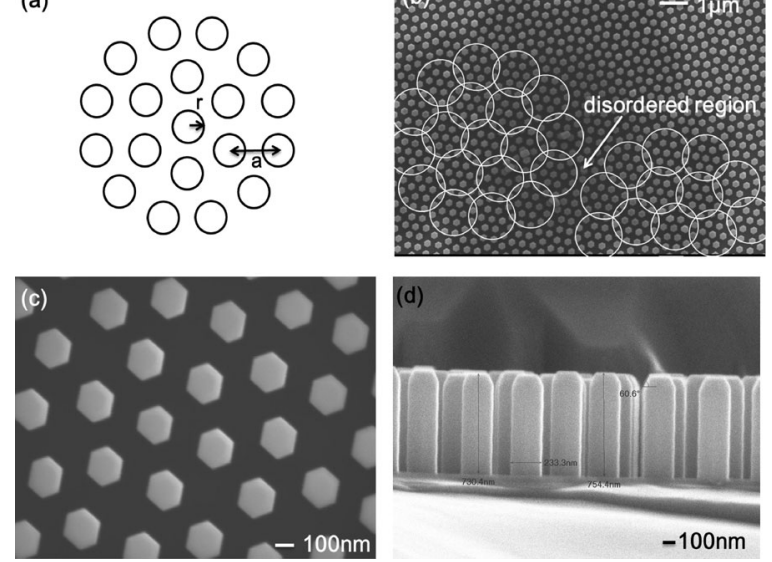

Fig. 1. (a) Scheme of GaN quasi-periodic nanopillars; $r$ and $a$ are, respectively, the radius and lattice constant of quasi-periodic nanopillars. (b) SEM plane view of a GaN quasi-periodic nanopillars. White circles indicate the unit cell of quasi-periodic nanopillars. (c) Enlarged SEM plane view and (d) crosssection image of GaN quasi-periodic nanopillars. The radius, lattice constant, and height of quasi-periodic nanopillars are estimated to be 134, 424, and $730 \mathrm{~nm}$, respectively.

100 torr. The flow sequence of $\mathrm{TMGa}$ and $\mathrm{NH}_{3}$ were introduced alternately with periods of 3 and $5 \mathrm{~s}$, respectively, for specific nanopillar growth mode. Fig. 1(a) shows the scheme of $\mathrm{GaN}$ quasi-periodic nanopillars structure. The scanning electron microscope (SEM) images of GaN quasi-periodic nanopillars are shown in Fig. 1(b)-(d). Fig. 1(b) shows a large area of quasiperiodic nanopillars and the white circles indicate the unit cell of quasi-periodic nanopillars. One white circle represents the scheme of the quasi-periodic nanopillar shown in Fig. 1(a). The radius, lattice constant, and height of quasi-periodic nanopillars were estimated to be 134,424 , and $730 \mathrm{~nm}$, respectively. The lattice constant of $\mathrm{GaN}$ quasi-periodic nanopillars, which are strongly related to the normalized frequency could decide the order of the resonant mode. The radius of GaN quasi-periodic nanopillars could adjust the band distribution and short range of photonic bandgap in the band structure. The optical pumping source used in the microphotoluminescence ( $\mu$-PL) system was the $355 \mathrm{~nm}$ pulse $\mathrm{Nd}: \mathrm{YVO}_{4}$ laser with a pulsewidth of $\sim 0.5 \mathrm{~ns}$ at a repetition rate of $1 \mathrm{kHz}$ and the experiments were all carried out at room temperature. The laser beam was pumped obliquely onto the sample with a spot size of about $20 \mu \mathrm{m}$. The PL signal was collected by a fiber with a $600 \mu \mathrm{m}$ core normal to the sample surface and fed into a spectrometer with a charge-coupled device (Jobin-Yvon IHR320 Spectrometer). The spectral resolution was about $0.1 \mathrm{~nm}$ for spectral output measurement.

\section{RESULT AND DISCUSSION}

Fig. 2 shows the pumping energy density versus the output characteristics curve of GaN quasi-periodic nanopillars. When the pumping energy density was increased around $40 \mathrm{~mJ} / \mathrm{cm}^{2}$, a clearly threshold condition could be observed. The spontaneous emission coupling factor $\beta$ of lasing mode in the GaN quasiperiodic nanopillars was also estimated by replotting Fig. 2 into a logarithm plot. We obtained the $\beta$ value of the lasing mode in

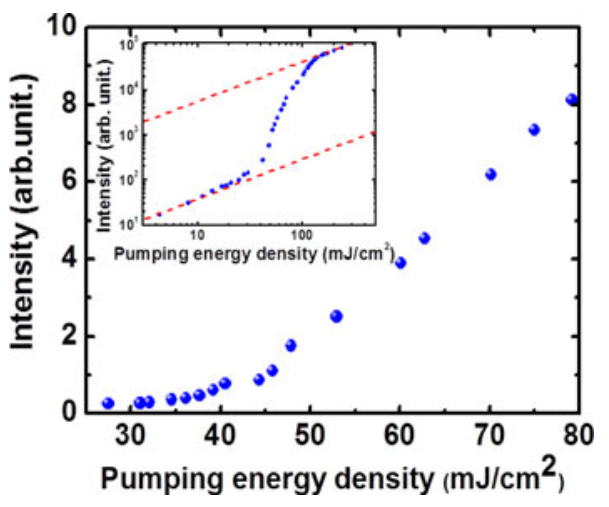

Fig. 2. Measured lasing emission intensity of the GaN quasi-periodic nanopillars versus the pumping energy density. The inset shows the log-log plot of measured lasing emission intensity versus the pumping energy density.

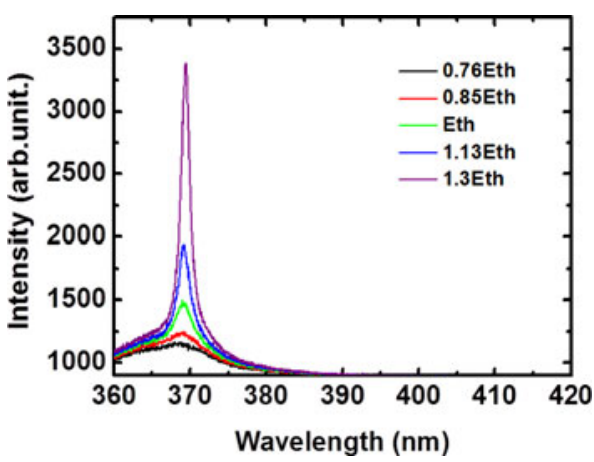

Fig. 3. Lasing spectra of the GaN quasi-periodic nanopillars as a function of the pumping energy density.

the GaN quasi-periodic nanopillars to be about $9.4 \times 10^{-3}$. The $\beta$ value was higher than the value obtained in the GaN-based PCSEL [8], which showed extended mode distribution characteristics over the whole PC region. Fig. 3 shows the lasing spectra of GaN quasi-periodic nanopillars. The linewidth was gradually narrowed to $1 \mathrm{~nm}$ when the input pumping power was increased. A dominated lasing peak that appeared above the threshold at $369 \mathrm{~nm}$ can be observed. From the emission spectra, the quality factor can be estimated to be about 170 when the GaN quasiperiodic nanopillars were operated in the transparent condition. The three-dimensional finite-element method (FEM) [23] was employed to calculate the quality factor of GaN quasi-periodic nanopillars. The quality factor was calculated to be 263 , which was somewhat higher than experimental results. It could be due to the imperfection of nanopillar shape causing the light scattering in real quasi-periodic nanopillars. The measured polarization of GaN quasi-periodic nanopillars is shown in Fig. 4. Moreover, the measured direction was aligned to the $\Gamma-K$ direction in the reciprocal space. The degree of polarization (DOP) is defined as $\left(I_{\max }-I_{\min }\right) /\left(I_{\max }+I_{\min }\right)$, where $I_{\max }$ and $I_{\min }$ are the maximum and the minimum intensity of lasing peak, respectively. The DOP was measured to be $55 \%$ when the GaN quasiperiodic nanopillars were above the threshold. Notice that our quasi-periodic nanopillars are the disordered structure between each unit cell, as shown in Fig. 1(b). The disordered nanopillars and imperfections may lead the diffraction light to feedback 


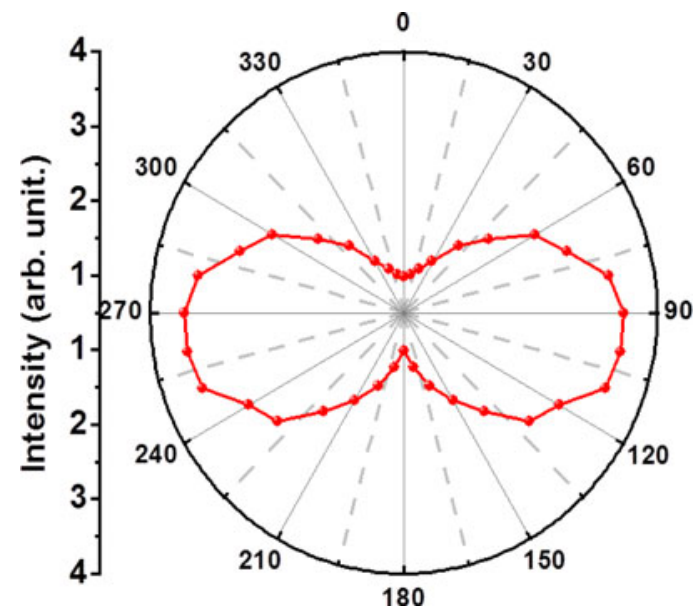

Fig. 4. DOP of the GaN quasi-periodic nanopillars. DOP is calculated to be about $55 \%$.

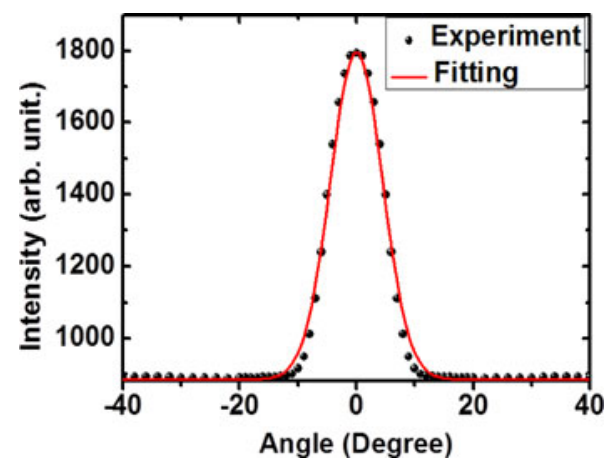

Fig. 5. Divergence angle of the GaN quasi-periodic nanopillars measured by angular-resolved micro-PL system. The divergence angle is calculated to be about $10.5^{\circ}$. The red line is the fitting curve.

not equally. However, the DOP would be stronger in specific directions such as $\Gamma-K$ and $\Gamma-M$ directions in the reciprocal space [8]. Fig. 5 represents the angle versus the output intensity for $\mathrm{GaN}$ quasi-periodic nanopillars. The angle resolution of the rotation stage was about $0.5^{\circ}$ for divergence angle measurement. The divergence angle that was estimated to be $10.5^{\circ}$, which is close to that of GaN-based VCSELs [24], reveals that the lasing spot size of the GaN quasi-periodic nanopillars should be few micrometers. Next, to observe the lasing-mode pattern of GaN quasi-periodic nanopillars, the near-field images were collected by $\times 100$ objective lens and measured by the beam view system. Fig. 6 shows the near-field image when it was below and above the threshold condition. As shown in Fig. 6(b), only one dominated lasing peak was observed in a localized region above the threshold condition, which was in sharp contrast to the large area lasing observed in the square-lattice or triangular-lattice band-edge mode GaN-based PCSELs [8]. The lasing spot of the $\mathrm{GaN}$ quasi-periodic nanopillars was estimated to be $3.6 \mu \mathrm{m}$ in diameter. Using the divergence angle equation $\theta=\lambda / \pi \omega_{0}$, where $\omega_{0}$ is the lasing spot size, $\lambda$ the lasing wavelength, and $\theta$ is the half of the divergence angle. We can calculate the laser spot size to be $4 \mu \mathrm{m}$ using the divergence angle obtained earlier, which is comparable to the measured divergence angle. It provides the
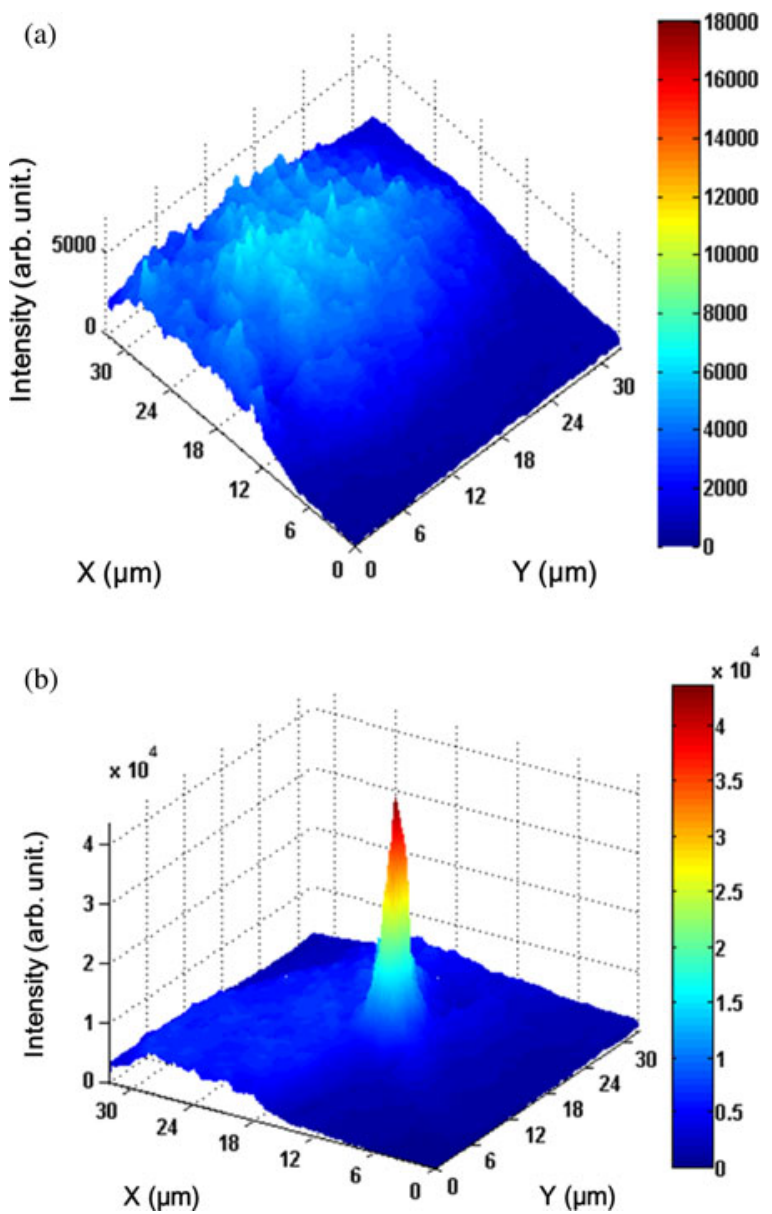

Fig. 6. Near-field images of GaN quasi-periodic nanopillars (a) below and (b) above the threshold condition.

strong evidence that the resonant mode in GaN quasi-periodic nanopillars could be highly localized in a specific region.

\section{Simulation Results}

To further understand the mechanism of the localized lasing mode in the GaN quasi-periodic nanopillars, multiple scattering method was employed to calculate the resonant wavelength and mode patterns in GaN quasi-periodic nanopillars. The detailed method and parameters in simulation were similar to the previous report [25]-[27]. Fig. 7(a) shows the mode pattern in the real and reciprocal space of the GaN quasi-periodic nanopillars. With the same resonant wavelength, the localized mode could be observed clearly in the center of the quasi-periodic nanopillars in Fig. 7(a). The calculated range of mode pattern is estimated about $3 \mu \mathrm{m}$ which is close to the localized lasing spot size in the near-field image. The reason of localized lasing mode could be due to the quasi-periodic nanopillars exist the short range of photonic bandgap, which supports the mode confinement in a localized region [18], [19], [21], [28]. It should be noted that the side peaks observed in Fig. 7(a) are due to the weak coupling $k$ vectors in reciprocal space. However, the side peaks are not appearing in Fig. 6(b). It could be due to the weak coupling $k$ vector would be scattered by the imperfect region, 

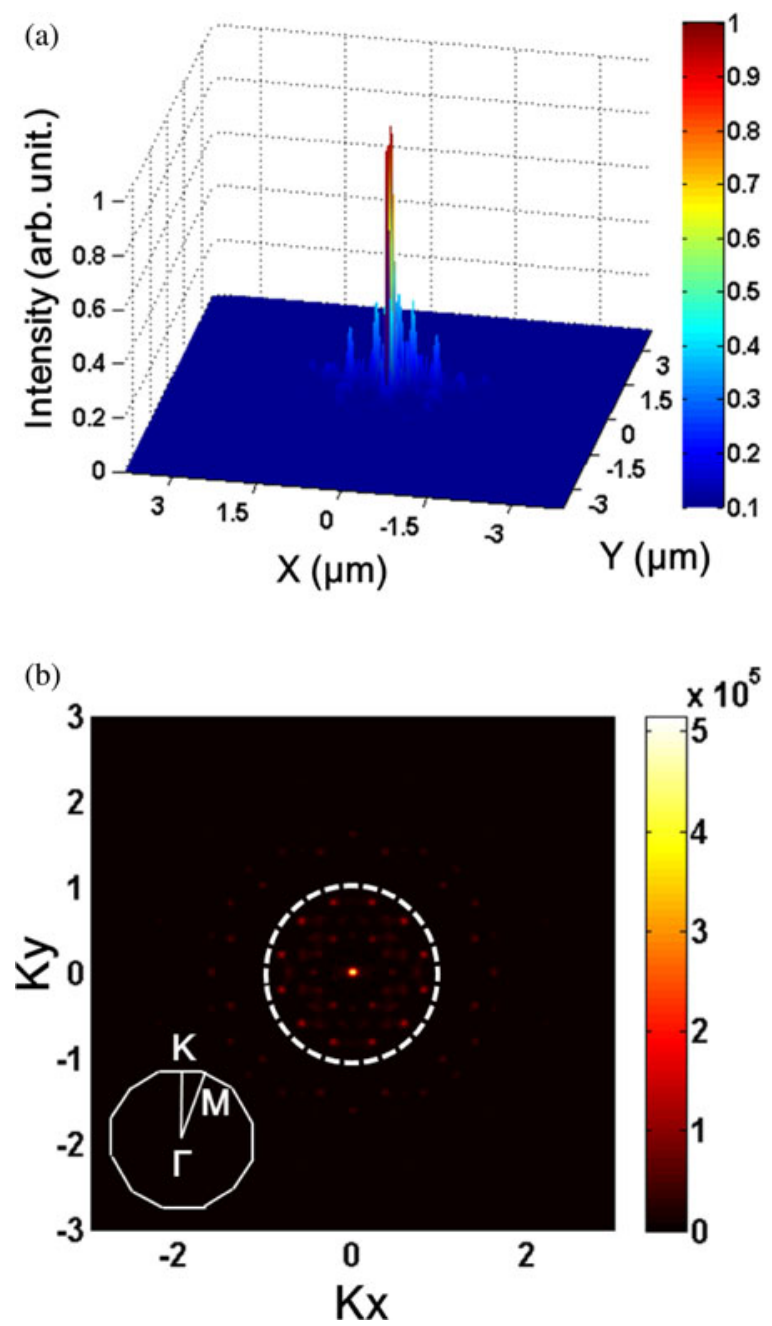

Fig. 7. (a) Calculated mode intensity pattern in the real space and (b) calculated magnetic field pattern in the reciprocal space of $\mathrm{GaN}$ quasi-periodic nanopillars using multiple scattering method. The white dashed circle is the light line in the quasi-periodic nanopillars. The inset shows the first Brillouin zone of the quasi-periodic nanopillars.

so the side peaks would not be observed in our beam view system. Fig. 7(b) shows the mode pattern in the reciprocal space of the quasi-periodic nanopillars structure. The white dashed line represents the light line of the quasi-periodic nanopillars and the bright spots represent the magnetic fields transferred by fast Fourier transform (FFT) [27]. It indicates that the localized lasing mode in quasi-periodic nanopillars is provided by highly symmetric multidirectional feedback mechanisms. The short range of photonic bandgap could be also confirmed by band structure using the PWE method [28]. By adjusting the super cell of quasi-periodic nanopillars [29] and using the effective index method [4], the parameters including confinement factor, filling factor, $\varepsilon_{a}$, and $\varepsilon_{b}$ were calculated to be $0.49,0.355$, 3.84 , and 4.67 , respectively. The definitions of $\varepsilon_{a}$ and $\varepsilon_{b}$ represent the effective dielectric constant of background material and nanopillars. Finally, the band structure of GaN quasi-periodic nanopillars can be obtained, as shown in Fig. 8. The short-range photonic bandgap ranging from 1.146 to 1.149 can be observed in the band structure. Compared with the experimental normal-

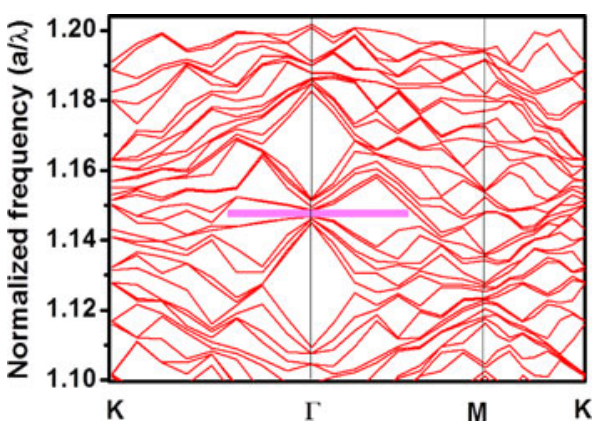

Fig. 8. Calculated band structure of GaN quasi-periodic nanopillars by PWE method. The magenta square represents the short range of photonic bandgap in band structure.

ized frequency of localized lasing mode, the normalized frequency is located at 1.148 , corresponding to the short range of photonic bandgap. The simulation results such as mode pattern in the real, reciprocal space, and band structure can provide the evidence of the localized mode in the GaN quasi-periodic nanopillars, which are comparable with the experimental results. The proper design of the quasi-periodic nanopillars and insertion of low refractive index layers would gradually increase the optical confinement of quasi-periodic nanopillars and reduce threshold condition to realize the low threshold lasing behavior in GaN quasi-periodic nanopillars in the future.

\section{SUMMARY}

In summary, GaN quasi-periodic nanopillars have been fabricated and characterized. The threshold energy density and lasing wavelength were measured to be $40 \mathrm{~mJ} / \mathrm{cm}^{2}$ and $369 \mathrm{~nm}$, respectively. The laser characteristics such as DOP and laser divergence angle were measured to be $55 \%$ and $10.5^{\circ}$, respectively. The near-field lasing spot and the spontaneous emission coupling factor were $3.6 \mu \mathrm{m}$ and $9.4 \times 10^{-3}$, respectively. Moreover, the localized lasing mode was observed in GaN quasi-periodic nanopillars and confirmed by the simulation results. We believe that the GaN quasi-periodic nanopillars have demonstrated an easy and cost-effective way to fabricate coherent light sources with localized modes, which would be helpful for photonic integration application in the near future.

\section{ACKNOWLEDGMENT}

The authors would like thank Prof. H. C. Kuo at National Chiao Tung University for his technical support and Nanocrystal Asia, Inc. for the GaN quasi-periodic nanopillar substrate support.

\section{REFERENCES}

[1] E. Yablonovitch, "Inhibited spontaneous emission in solid-state physics and electronics," Phys. Rev. Lett., vol. 58, pp. 2059-2062, 1987.

[2] S. John, "Strong localization of photons in certain disordered dielectric superlattices," Phys. Rev. Lett., vol. 58, pp. 2486-2489, 1987.

[3] M. Meier, A. Mekis, A. Dodabalapur, A. Timko, R. E. Slusher, J. D. Joannopoulos, and O. Nalamasu, "Laser action from twodimensional distributed feedback in photonic crystals," Appl. Phys. Lett., vol. 74, p. 7, 1999. 
[4] M. Imada, A. Chutinan, S. Noda,, and M. Mochizuki, "Multidirectionally distributed feedback photonic crystal lasers," Phys. Rev. B, Condens. Matter, vol. 65, pp. 195306-1-195306-8, 2002.

[5] E Matioli, E. Rangel, M. Iza, B. Fleury, N. Pfaff, J. Speck, E. Hu, and C. Weisbuch, "High extraction efficiency light-emitting diodes based on embedded air-gap photonic-crystals," Appl. Phys. Lett., vol. 96, pp. 031108-1-031108-3, 2010.

[6] I. Vurgaftman and J. Meyer, "Design optimization for high-brightness surface-emitting photonic-crystal distributed-feedback lasers," IEEE $J$. Quantum Electron., vol. 39, no. 6, pp. 689-700, Jun. 2003.

[7] H. Matsubara, S. Yoshimoto, H. Saito, Y. Jianglin, Y. Tanaka, and S. Noda, "GaN photonic-crystal surface-emitting laser at blue-violet wavelengths," Science, vol. 319, pp. 445-447, 2008

[8] T. C. Lu, S. W. Chen, L. F. Lin, T. T. Kao, C. C. Kao, P. Yu, H. C. Kuo, S. C. Wang,, and S. H. Fan, "GaN two-dimensional surface-emitting photonic crystal lasers with AlN/GaN distributed Bragg reflector," Appl. Phys. Lett., vol. 92, pp. 011129-1-011129-3, 2008.

[9] S. Kawashima, T. Kawashima, Y. Nagatomo, Y. Hori, H. Iwase, T. Uchida, K. Hoshino, A. Numata, and M. Uchida, "GaN surface-emitting laser with two-dimensional photonic crystal acting as distributed-feedback grating and optical cladding," Appl. Phys. Lett., vol. 97, pp. 251112-1-251112-3, 2010.

[10] M Kim, C. S. Kim, W. W. Bewley, J. R. Lindle, C. L. Canedy, I. Vurgaftman,, and J. R. Meyer, "Surface-emitting photonic-crystal distributed-feedback laser for the midinfrared," Appl. Phys. Lett., vol. 88, pp. 191105-1-191105-3, 2006.

[11] L. Sirigu, R. Terazzi, I. Amanti, M. Giovannini, J. Faist, A. Dunbar, and R. Houdré, "Terahertz quantum cascade lasers based on two-dimensional photonic crystal resonators," Opt. Exp., vol. 16, pp. 5206-5217, 2008.

[12] O. Painter, R. K. Lee, A. Scherer, A. Yariv, J. D. O'Brien, P. D. Dapkus, and I. Kim, "Two-dimensional photonic band-gap defect mode laser," Science, vol. 284, pp. 1819-1821, 1999.

[13] H. G. Park, S. H. Kim, S. H. Kwon, Y. G. Ju, J. K. Yang, J. H. Baek, S. B. Kim, and Y. H. Lee, "Electrically driven single-cell photonic crystal laser," Science, vol. 305, pp. 1444-1447, 2005.

[14] C. Meiera, K. Hennessy, E. D. Haberer, R. Sharma, Y. S. Choi, K. McGroddy, S. Keller, S. P. DenBaars, S. Nakamura, and E. L. Hu, "Visible resonant modes in GaN photonic crystal membrane cavities," Appl. Phys. Lett., vol. 88, pp. 031111-1-031111-3, 2006.

[15] M. Arita, S. Ishida, S. Kako, S. Iwamoto, and Y. Arakawa, "AlN airbridge photonic crystal nanocavities demonstrating high quality factor," Appl. Phys. Lett., vol. 91, pp. 051106-1-051106-3, 2007.

[16] M. E. Zoorob, M. B. D. Charlton, G. J. Parker, J. J. Baumberg,, and M. C. Natti, "Complete photonic bandgaps in 12-fold symmetric quasicrystals," Nature, vol. 404, pp. 740-743, 2000.

[17] Y. Q. Wang, X. Y. Hu, X. S. Xu, B. Y. Cheng,, and D. Z. Zhang, "Localized modes in defect-free dodecagonal quasiperiodic photonic crystals," Phys. Rev. B, Condens. Matter, vol. 68, pp. 165106-1-165106-4, 2003.

[18] M Notomi, H. Suzuki, T. Tamamura,, and K. Edagawa, "Lasing action due to the two-dimensional quasiperiodicity of photonic quasicrystals with a penrose lattice," Phy. Rev. Lett., vol. 92, pp. 123906-1-123906-4, 2004.

[19] A. D. Villa, S. Enoch, G. Tayeb, V. Pierro, V. Galdi,, and F. Capolino, "Band gap formation and multiple scattering in photonic quasicrystals with a penrose-type lattice," Phy. Rev. Lett., vol. 94, pp. 183903-1-183903-4, 2005.

[20] K. Mnaymneh and R. Gauthier, "Mode localization and band-gap formation in defect-free photonic quasicrystals," Opt. Exp., vol. 15, pp. 50895099, 2007

[21] T. Priya Rose, E. Di Gennaro, G. Abbate, and A. Andreone, "Isotropic properties of the photonic band gap in quasicrystals with low-index contrast," Phy. Rev. B, Condens. Matter, vol. 84, pp. 125111-1-125111-7, 2011.

[22] L. Levi, M. Rechtsman, B. Freedman, T. Schwartz, O. Manela,, and M. Segev, "Disorder-enhanced transport in photonic quasicrystals," Science, vol. 332, pp. 1541-1544, 2011.

[23] W. J. Kim and J. D. O'Brien, "Optimization of a two-dimensional photonic-crystal waveguide branch by simulated annealing and the finiteelement method," J. Opt. Soc. Amer. B, Opt. Phys., vol. 21, pp. 289-295, 2004.

[24] T. C. Lu, S. W. Chen, T. T. Wu, P. M. Tu, C. K. Chen, C. H. Chen, Z. Y. Li, H. C. Kuo,, and S. C. Wang, "Continuous wave operation of current injected GaN vertical cavity surface emitting lasers at room temperature," Appl. Phys. Lett., vol. 97, pp. 071114-1-071114-3, 2010.

[25] S. Nojima, "Theoretical analysis of feedback mechanisms of twodimensional finite-sized photonic-crystal lasers," J. Appl. Phys., vol. 98, pp. 043102-1-043102-9, 2005.
[26] S. Nojima, "Optical-gain enhancement in two-dimensional active photonic crystals," J. Appl. Phys., vol. 90, pp. 545-551, 2001.

[27] P. S. Weng, T. T. Wu, and T. C. Lu, "Study of band-edge modes in $\mathrm{GaN}$ photonic crystal surface-emitting lasers by the multiple-scattering method," IEEE J. Sel. Topics Quantum Electron., vol. 18, no. 6, pp. 16291635, Nov./Dec. 2012.

[28] K. Sakoda, K. Ohtaka, and T. Ueta, "Low-threshold laser oscillation due to group-velocity anomaly peculiar to two- and three-dimensional photonic crystals," Opt. Exp., vol. 4, pp. 481-489, 1999.

[29] B. P. Hiett, D. H. Beckett, S. J. Cox, J. M. Generowicz, M. Molinari, and K. S. Thomas, "Photonic band gaps in 12-fold symmetric quasicrystals," J. Mater. Sci. Mater. Electron., vol. 14, pp. 413-416, 2003.

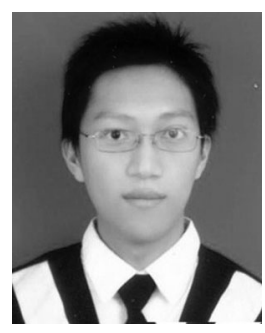

Tzeng-Tsong Wu (S'12) received the B.S. degree in electrical engineering and the M.S. degree in optoelectronic sciences from the National Taiwan Ocean University (NTOU), Keelung, Taiwan, in 2007 and 2009 , respectively. He is currently working toward the Ph.D. degree in electro-optical engineering in the Department of Photonics, Institute of ElectroOptical Engineering, National Chiao Tung University (NCTU), Hsinchu, Taiwan

In 2009, he joined the Semiconductor Laser Technology Laboratory, NCTU, where he was involved in research on III-V semiconductor materials for semiconductor lasers under the instruction of Prof. T.-C. Lu and Prof. S.-C. Wang. His current research interests include GaN vertical-cavity surface-emitting laser (VCSEL) and photonic crystal surface-emitting laser (PCSEL).

Chih-Cheng Chen is currently working toward the M.S. degree in the Department of Photonics, National Chiao Tung University (NCTU), Hsinchu, Taiwan.

In 2011, he joined the Semiconductor Laser Technology Laboratory, NCTU, under supervision of Prof. Tien-Chang Lu. His current research interests include modeling, simulation, and processing of $\mathrm{GaN}$ photonic crystal surface-emitting laser (PCSEL).

Hao-Wen Chen is currently working toward the M.S. degree in the Department of Photonics, National Chiao Tung University (NCTU), Hsinchu, Taiwan.

In 2011, she joined the Semiconductor Laser Technology Laboratory, NCTU, under supervision of Prof. Tien-Chang Lu. Her current research interests include modeling, simulation, and processing of GaN photonic crystal laser.

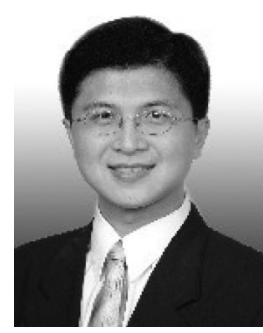

Tien-Chang Lu (M'07-SM'12) received the B.S. degree in electrical engineering from the National Taiwan University, Taipei, Taiwan, in 1995, the M.S. degree in electrical engineering from the University of Southern California, Los Angeles, USA, in 1998, and the Ph.D. degree in electrical engineering and computer science from the National Chiao Tung University (NCTU), Hsinchu, Taiwan, in 2004.

In 2004, he was a Manager with in the Epitaxy Department, Union Optronics Corporation. Since August 2005, he has been a member of the faculty in the Department of Photonics, NCTU. His research work included the design, epitaxial growth, process, and characterization of optoelectronic devices. He has been involved in the research on low-pressure metal-organic chemical vapor deposition epitaxial technique associated with various material systems as well as the corresponding process skills. His current research interests include structure design and simulations for optoelectronic devices using computer-aided software. He has authored or coauthored more than 180 internal journal papers.

Dr. Lu is a recipient of the Exploration Research Award of Pan Wen Yuan Foundation 2007, the Excellent Young Electronic Engineer Award 2008, the Young Optical Engineer Award 2010, the MOC'11 Contribution Award 2011, and Dr. Ta-Yu Wu's Memorial Award 2012. 


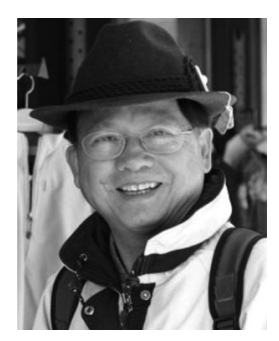

Shing-Chung Wang (M'79-SM'03-LM'07) received the B.S. degree in electrical engineering from the National Taiwan University, Taipei, Taiwan, the M.S. degree in electrical engineering from the National Tohoku University, Sendai, Japan, and the $\mathrm{Ph} . \mathrm{D}$. degree in electrical engineering from the Stanford University, Stanford, CA, USA, in 1971.

He was a member of the faculty at the National Chiao Tung University (NCTU), Hsinchu, Taiwan (from 1965 to 1967), a Research Associate at Stanford University (from 1971 to 1974), a Senior Research Scientist at Xerox Corporation (from 1974 to 1985), and a Consulting Scientist at Lockheed-Martin Palo Alto Research Laboratories (from 1985 to 1995). Since 1995, he has been a member of the faculty with the Institute of Electro-Optical Engineering, NCTU. He has authored or coauthored more than 160 publications. His current research interests include semiconductor lasers, vertical-cavity surface-emitting lasers, blue and UV lasers, quantum-confined optoelectronic structures, optoelectronic materials, diode-pumped lasers, and semiconductor laser applications.

Prof. Wang is a Fellow of the Optical Society of America and the recipient of the Outstanding Scholar Award from the Foundation for the Advancement of Outstanding Scholarship.

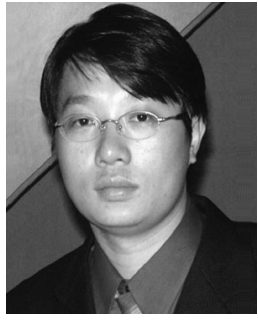

optoelectronic devices.
Cheng-Huang Kuo was born in Tainan, Taiwan, in 1976. He received the M.S. degree from the Department of Physics, National Central University (NCU), Jhongli, Taiwan, in 2001, and the Ph.D. degree from the Institute of Microelectronics, National Cheng Kung University, Tainan, Taiwan, in 2004.

$\mathrm{He}$ is currently an Associate Professor with the Institute of Lighting and Energy Photonics, National Chiao Tung University, Tainan, Taiwan. His current research interests include semiconductor physics and 